\title{
Self-sensing Control of the Externally-Excited Synchronous Machine for Electric Vehicle Traction Application
}

\author{
Mohamad Koteich $^{1}$, IEEE Member, Amir Messali ${ }^{2}$, Student Member, Simon Daurelle ${ }^{2}$, Student Member
}

\begin{abstract}
This paper studies the possibility of removing the position sensor from electric vehicles (EVs) powertrains that employ the externally-excited synchronous machine (EESM) for traction. Three position estimation approaches are studied: back-electromotive-force integration, state-observer and highfrequency voltage injection approaches. Theoretical background is presented, a comparative performance analysis is performed, and experimental results on a $65-\mathrm{kW}$ EESM drive test bench are shown. Some requirements for $\mathrm{EV}$ powertrains are emphasized.
\end{abstract}

\section{INTRODUCTION}

The electric vehicle (EV) market is expected to grow increasingly in the next decade. Most of nowadays EVs use inset permanent-magnet synchronous machine (IPMSM) drive for traction. The main challenge of the IPMSM in the automotive industry is the dependence on the rare-earth market. Several alternative solutions have been studied in order to remove magnets from EV drives without increasing their volume and their mass [1]. One potential alternative is the externally-excited synchronous machine (EESM) which is being successfully used in Renault Zoé, Fluence and Kangoo EVs.

The present paper studies the possibility of removing the position sensor from the EESM drive system, using selfsensing control techniques [2], in order to reduce the cost and increase both the reliability and the mechanical robustness of the drive. Compared to the IPMSM, very few papers have studied the self-sensing capabilities of the EESM [3]-[7], especially that several designs of the latter have been proposed: if the Zoé traction drive is taken as example, there exist at least 3 different EESM powertrains, the first two generations are manufactured by Continental, and the latter ones are Renault in-house manufactured machines.

This paper is aimed at presenting a primary study of the feasibility of self-sensing control for EESM traction drives. Three broad approaches for position estimation are tested and compared: 1) back-electromotive force (EMF) integration approach [8], presented in Section III, 2) state-observer approach [6], in Section IV, and 3) high-frequency (HF) injection approach [9], in Section V. The performance of the first approach are presented for medium and high speed. The limitations of the second approach are analyzed. The third approach has two major advantages over the first two ones: 1) it works stably at low speed and standstill and 2) it does not require the knowledge of any machine parameter.

Experimental tests have been performed on a 4-poles, 65$\mathrm{kW}$ EESM, the results are presented in Section VI.

\footnotetext{
1 Groupe Renault, Technocentre, Guyancourt, France

${ }^{2}$ Ecole Centrale Nantes, LS2N Laboratory, Nantes, France
}

\section{EESM MATHEMATICAL MODEL}

The schematic representation of the EESM is shown on Fig. 1: a three-phase stator, with an externally DC-excited winding in the rotor [6].

\section{A. Notations}

The complex space vector notation is used to model the revolving electromagnetic stator quantities [10]. Let $\underline{x}_{s}^{r}$ be a stator quantity in the rotor reference frame, it can be expressed as

$$
\underline{x}_{s}^{r}=\underline{x}_{s}^{s} e^{-j \theta}
$$

or equivalently

$$
x_{s d}+j x_{s q}=\left(x_{s \alpha}+j x_{s \beta}\right)(\cos \theta-j \sin \theta)
$$

Subscripts $s$ and $f$ stand for stator and rotor (field) quantities respectively, whereas superscripts $s$ and $r$ tell whether the quantity is expressed in the stator $(\alpha \beta)$ or the rotor $(d q)$ reference frame. $\theta$ is the angle between the stator and the rotor reference frames. The fluxes, currents, voltages and back EMFs are denoted by $\psi, i, v$ and $e$, respectively. The inductance and the resistance of a given circuit are denoted by $L$ and $R$ respectively, and $M$ denotes the mutual inductance between the stator and rotor circuits.

\section{B. Stator equations}

The stator voltage equation in the stator coordinates can be written as:

$$
\underline{v}_{s}^{s}=R_{s} \underline{i}_{s}^{s}+\frac{d \underline{\psi}_{s}^{s}}{d t}
$$

In the rotor reference frame, the previous equation becomes:

$$
\underline{v}_{s}^{r}=R_{s} \underline{i}_{s}^{r}+\frac{d \underline{\psi}_{s}^{r}}{d t}+j \omega \underline{\psi}_{s}^{r}
$$

Neglecting the cross-saturation inductances, the stator flux can be expressed in the rotor coordinates as:

$$
\begin{aligned}
\underline{\psi}_{s}^{r} & =\frac{L_{d}+L_{q}}{2} \underline{i}_{s}^{r}+\frac{L_{d}-L_{q}}{2} \underline{i}_{s}^{r *}+M_{f} i_{f} \\
& =L_{0} \underline{i}_{s}^{r}+L_{2} \underline{i}_{s}^{r *}+M_{f} i_{f}
\end{aligned}
$$

where $\underline{i}_{s}^{r *}$ denotes the complex conjugate of $\underline{i}_{s}^{r}$.

\section{Rotor equations}

The rotor scalar equations are expressed in the rotor reference frame. The voltage equation is:

$$
v_{f}=R_{f} i_{f}+\frac{d \psi_{f}}{d t}
$$

with

$$
\psi_{f}=L_{f} i_{f}+M_{f} i_{s d}
$$




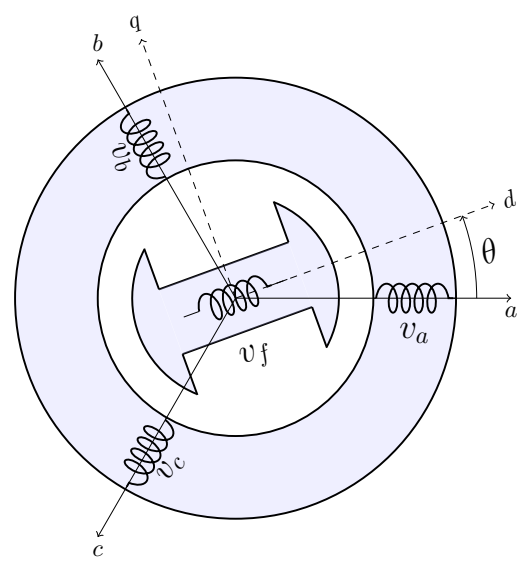

Fig. 1: The EESM schematic representation

\section{Equivalent-Flux BASED estimation}

This section describes a unified position estimator for AC machines. It is designed by merging of the contributions of several papers including [8], [11]-[15]. The following estimator consists of two parts: stator flux estimation based on a programmable statically-compensated (PSC) low-pass filter (LPF), and rotor flux position estimation based on the Equivalent Flux, or Active Flux, concept described below.

\section{A. Stator flux estimation}

The stator flux vector can be estimated by integrating the back-EMF $\left(\underline{e}_{s}^{s}\right)$ :

$$
\underline{\psi}_{s}^{s}=\int\left(\underline{v}_{s}^{s}-R_{s} \underline{i}_{s}^{s}\right) d t=\int \underline{e}_{s}^{s} d t
$$

where $\underline{v}_{s}^{s}$ is the command voltage estimated using an inverter model, $R_{s}$ is considered to be fairly accurately known, and $\underline{i}_{s}^{s}$ is the measured stator current.

The implementation of a pure integrator is prone to drift problems due to inverter nonlinearities, current measurement noises, unbalanced gains and DC offset. Furthermore, an initial condition error would result in a DC-offset in the integrator output. To overcome these problems, various algorithms have been reported in the literature, including adaptive flux observers [13], which require the knowledge of the machine inductances. Nevertheless, it can be argued that if the (openloop) integration function is optimized, the use of adaptive (closed-loop) estimation structure would not be easily justifiable. Thus, an optimized modified integration algorithm is designed for stator flux estimation.

To remove the error due to the DC-offset at the input of the integrator, a high-pass filter, with a corner frequency $\omega_{c}$, is implemented in series with the input of the pure integrator. This results in a low-pass filter (LPF) approximated integrator:

$$
\frac{\widehat{\Psi}_{s}(s)}{\mathbf{e}_{s}(s)}=\frac{1}{s} \cdot \frac{s}{s+\omega_{c}}=\frac{1}{s+\omega_{c}}
$$

The filter generates both magnitude distortion and phase lag, which can be compensated for, in steady-state, by multiplying the filter by the following function, in order to bring the LPF response closer to the pure integrator response [11]:

$$
\frac{j \omega_{s}+\omega_{c}}{j \omega_{s}}=1-j \frac{\omega_{c}}{\omega_{s}}
$$

Furthermore, the tunning of the filter cut-off frequency is a trade-off between the offset rejection dynamics and the steadystate accuracy: for example, higher corner frequency $\omega_{c}$ ensures faster DC-offset rejection, however, it introduces higher distortion in the output signal due to increasing attenuation and phase lag. Therefore, an adaptive corner frequency tuning should be adopted: $\omega_{c}$ is chosen in a way to be dependent on the stator angular frequency $\omega_{s}$ as follows [8]:

$$
\omega_{c}=\lambda\left|\omega_{s}\right|
$$

where $\lambda$ is a positive real number smaller than one. At low speed, $\lambda$ can be tuned to a low value, e.g. 0.1, whereas for higher speeds, it can take higher values. In this case, the timeconstant of the LPF, $1 /\left(\lambda\left|\omega_{s}\right|\right)$, is decreased with the increase of the stator frequency. The time-domain expression of such a PSC-LPF is [8]:

$$
\underline{\hat{\psi}}_{s}^{s}=\int\left(-\lambda\left|\omega_{s}\right| \underline{\hat{\psi}}_{s}^{s}+\left[1-j \lambda \operatorname{sign}\left(\omega_{s}\right)\right] \underline{e}_{s}^{s}\right) d t
$$

The performance of the PSC-LPF depends on the stator resistance, the accuracy of the inverter model, and the choice of $\lambda$. Furthermore, it heavily relies on the accuracy of the stator flux angular frequency $\left(\omega_{s}\right)$ estimate. A PLL-based estimation scheme, applied to the command voltage, is implemented to estimate the stator angular frequency [12], as shown in Fig. 2.

\section{B. Equivalent-flux concept and position estimation}

The stator back-EMF integration is valid for all AC machines, since they all have the same voltage structure. The rotor position estimation based on the stator flux relies on the interaction between the stator and the rotor fields, which is dependent on the rotor structure. Nevertheless, a unified flux model can be developed in view of rotor field-oriented control of AC drives, by introducing the Equivalent Flux concept $\psi_{e q}$ and an equivalent stator inductance $L_{e q}$ [14]:

$$
\begin{aligned}
\underline{\psi}_{s}^{s} & =L_{e q} \underline{i}_{s}^{s}+\underline{\psi}_{e q}^{s} \\
\underline{\psi}_{e q}^{s} & =\psi_{e q} e^{j \theta}
\end{aligned}
$$

with $L_{e q}=L_{q}$ for synchronous machines and $L_{e q}=\sigma L_{s}$ for inductions machines (IMs), and the equivalent flux $\psi_{e q}$ is expressed for the EESM, IPMSM and the IM as the following:

$$
\begin{array}{lll}
\text { EESM } & : & \psi_{e q}=\left(L_{d}-L_{q}\right) i_{s d}+M_{f} i_{f} \\
\text { IPMSM } & : & \psi_{e q}=\left(L_{d}-L_{q}\right) i_{s d}+\psi_{r} \\
\text { IM } & : & \psi_{e q}=k_{r} \psi_{r d}
\end{array}
$$

The rotor position of the EESM can be estimated from the stator flux by evaluating the phase angle of the equivalent flux vector using the arctangent function for example.

The complex signal flow of the equivalent-flux-based position estimator is shown on Fig. 2. 


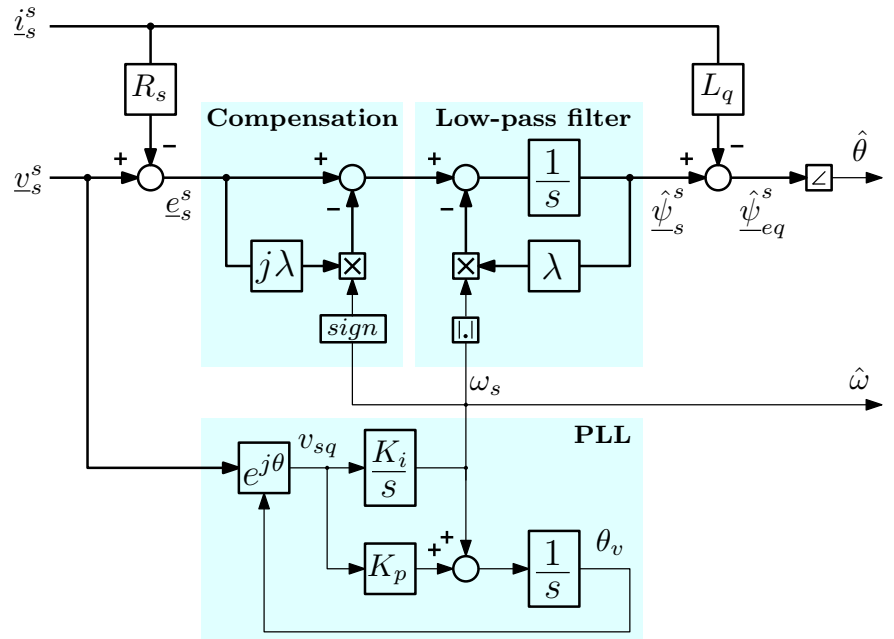

Fig. 2: Equivalent-flux based Estimator

\section{State-observer based estimation}

The state-space model of the EESM can be deduced from Section II, by incorporating the mechanical model equations [6]. A state-observer, such as the extended Kalman filter (EKF) can be designed to estimate the rotor position and speed based on the stator and rotor currents measurement. Nevertheless, a state observer requires the system to satisfy the so-called observability conditions, in order to ensure stable and accurate estimation. The observability of the EESM is studied in [6], where it is shown that at standstill, the observability is not guaranteed, unless some varying (high-frequency) voltage is injected in the machine.

According to the authors experience, a full-order observer, such as the EKF, is not a practical solution for EV's EESM self-sensing control, for the following reasons:

- Observer-based estimation fails at very low-speed, unless an $\mathrm{HF}$ voltage is injected. In the case of the studied EESM, the injection in the rotor winding is not helpful because of the low bandwidth of the rotor circuit. A low amplitude HF signal will be filtered if injected to the rotor, and lower frequency signal would generate torque ripple. On the other hand, if the HF signal is to be injected in the stator, then HFI techniques would be preferred thanks to their robustness and simplicity.

- The model does not only rely on the knowledge of $L_{d}$ and $L_{q}$, but also on $L_{f}$ and $M_{f}$. These inductances cannot be accurately known due to nonlinear saturation phenomenon that occur in the machine. Therefore, apart from observability problems at low speed, a state-observer, such as the EKF, would fail to accurately estimate the position over the whole operating range, due to the absence of an accurate linearized model.

- the EKF implementation requires high computational burden.

As a conclusion, state observers that rely on the knowledge of the inductances are less likely to work on the EESM, where

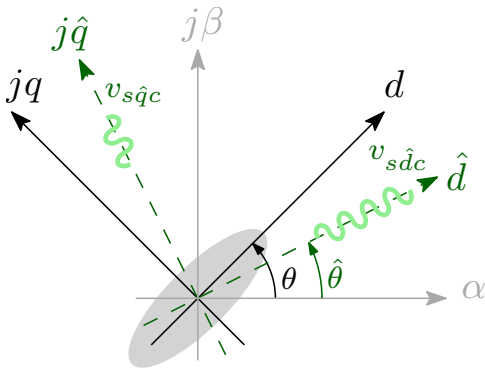

Injected Voltage

$v_{s \hat{d} c}=V_{c} \cos \omega_{c} t$

$v_{s \hat{q} c}=\frac{\hat{\omega}}{\omega_{c}} V_{c} \sin \omega_{c} t$

Resulting Flux

$\psi_{s \hat{d} c}=\frac{V_{c}}{\omega_{c}} \sin \omega_{c} t$

$\psi_{s \hat{q} c}=0$

Fig. 3: HF voltage injection principle.

magnetic saturation phenomena are more complex than the IPMSM. More robust methods are to be sought.

\section{Pulsating HF InJECTION BASEd ESTIMATION}

The pulsating HF injection (HFI) position estimation has been proposed for the IPMSM by Corley and Lorenz [9]. It is based on the property of the $d$ - and $q$ - axes flux being decoupled: if the following HF voltage vector, at $\omega_{c}$ angular frequency $\left(\omega_{c}>>\omega_{s}\right)$

$$
\underline{v}_{s c}^{\hat{r}}=V_{c} \cos \left(\omega_{c} t\right)+j V_{c} \frac{\hat{\omega}_{s}}{\omega_{c}} \sin \left(\omega_{c} t\right)
$$

is injected to the drive, in the estimated rotor reference frame (Fig. 3), it is expected to induce HF current only on the estimated $d$-axis. The measured HF current through the estimated $q$-axis is driven to zero via a PI mechanism in order to make the estimated position track the actual position. The HF resistive drop voltage can be neglected. The induced HF flux in the estimated position coordinates is

$$
\underline{\psi}_{s c}^{\hat{r}}=\frac{V_{c}}{\omega_{c}} \sin \left(\omega_{c} t\right)
$$

it can be expressed in the stationary coordinates as follows:

$$
\underline{\psi}_{s c}^{s}=\frac{V_{c}}{\omega_{c}} \sin \left(\omega_{c} t\right) e^{j \hat{\theta}}
$$

The induced HF current in the stationary coordinates can be written as (by inverting equation 4 and transforming it into the stator reference frame):

$$
\begin{aligned}
\underline{i}_{s c}^{s} & =\frac{1}{L_{0}^{2}-L_{2}^{2}}\left(L_{0} \underline{\psi}_{s c}^{s}-L_{2} \underline{\psi}_{s c}^{s *} e^{j 2 \theta}\right) \\
& =I_{c p} e^{j \hat{\theta}} \sin \left(\omega_{c} t\right)-I_{c n} e^{j(2 \theta-\hat{\theta})} \sin \left(\omega_{c} t\right)
\end{aligned}
$$

$I_{c p}$ and $I_{c n}$ denote the maximum magnitude positive- and negative-sequence components of the HF currents respectively:

$$
\begin{aligned}
I_{c p} & =\frac{V_{c}}{\omega_{c}} \frac{L_{0}}{L_{0}^{2}-L_{2}^{2}}=\frac{V_{c}}{\omega_{c}} \frac{L_{0}}{L_{d} L_{q}} \\
I_{c n} & =\frac{V_{c}}{\omega_{c}} \frac{L_{2}}{L_{0}^{2}-L_{2}^{2}}=\frac{V_{c}}{\omega_{c}} \frac{L_{2}}{L_{d} L_{q}}
\end{aligned}
$$

The HF $q$-axis current component is extracted using a first order high-pass filter (HPF), and the following position estimation error signal, $\varepsilon$, is evaluated by low-pass filtering this HF component multiplied by $\sin \left(\omega_{c} t\right)$ :

$$
\varepsilon=L P F\left[\sin \left(\omega_{c} t\right) \mathcal{I} m\left(\underline{i}_{s c}^{s} e^{-j \hat{\theta}}\right)\right]
$$




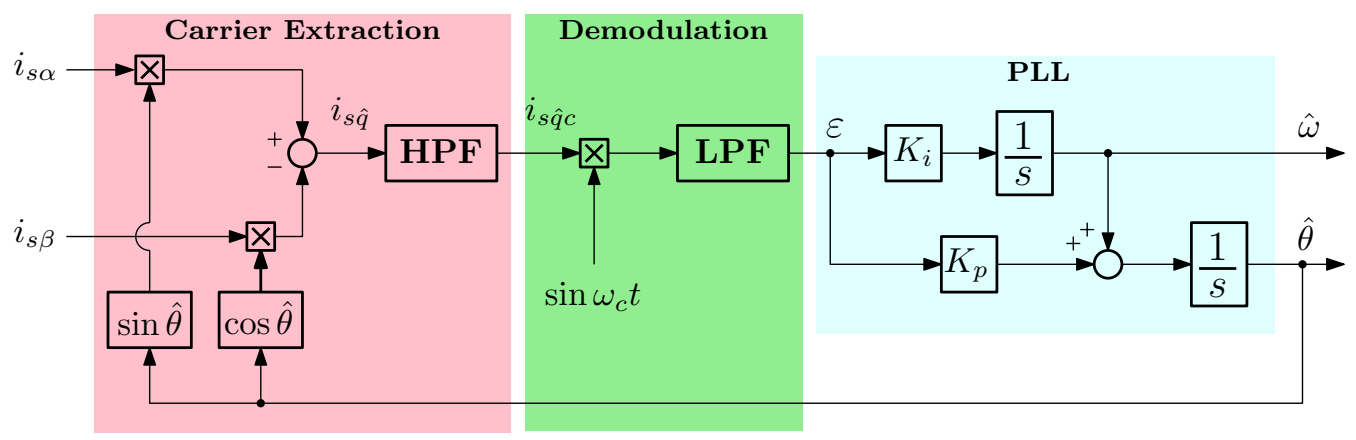

Fig. 4: Demodulation signal processing for HFI sensorless control

which finally yields:

$$
\varepsilon=\frac{I_{c n}}{2} \sin 2(\theta-\hat{\theta}) \approx I_{c n}(\theta-\hat{\theta})
$$

This signal is then fed to a PLL tracking mechanism that outputs the position. The demodulation process is illustrated in Fig. 4. For more details about the PLL tuning refer to [16].

The Pulsating HFI is also a generic estimation technique that can be applied to AC drives. It only required a magnetic anisotropy in the flux path. In the case of the EESM, the stator HF current can generate a rotor HF current due to the coupling between the two circuits. Nevertheless, as mentioned earlier, the rotor bandwidth is much lower than the stator one for the studied EESM, and the HF signal is chosen in a way to be filtered by the rotor circuit.

\section{EXPERIMENTAL RESULTS}

This section presents the results of the experimental tests that have been performed on the 4-poles $65-\mathrm{kW}$ Renault manufactured EESM drive that is used on the Zoé electric car. The drive is operated in sensorless configuration, the position sensor is used for comparison. A $10 \mathrm{kHz}$ switching frequency PWM voltage source inverter is used, with a 400 Volt DC bus.

\section{A. Flux-based position estimation}

The Equivalent-flux-based position estimator presented in Section III has been tested under self-sensing control configuration, for a speed ramp, from 1000 to $9000 \mathrm{rpm}$, with no- and full-load. The results are shown on Fig. 7. The value of $L_{q}$ is generated based on a look-up table with two inputs (for simplicity): the estimated speed and estimated torque. The look-up table is filled based on experimental tests. Other techniques can be used for $L_{q}$ identification, such as polynomial identification using the stator current as input. To the best of the knowledge of the authors, the adaptive, robust estimation of $L_{q}$ is still an open problem, and no promising solutions have been proposed yet.

The position estimation error depends mainly on the values of $L_{q}$. Higher accuracy and higher precision look-up table is needed to ensure better estimation quality. Position estimation based on flux estimation is not stable at low speed, due to the low amplitude range of the back-EMF.
The speed estimation using the stator voltage PLL seem to be fairly accurate. Its dynamical performance can be further improved by fine tuning the PLL or by incorporating the mechanical model and the torque request.

\section{B. Kalman Filter based position estimation}

The extended Kalman filter (EKF) algorithm shown in Fig. 5 has been tested, using the stationary $\alpha \beta$ electromechanical model of the EESM [6]. System linearization matrices and matrix inversion calculation had been done analytically, off-line, in order to reduce computational burden. The HF injection in the rotor winding proposed in [6] has been implemented. Fig. 6 shows that at very low speed, when a HF current is injected to the rotor winding, the position estimation error is around zero, which is not the case when no HF current is injected. This is consistent with the observability analysis results presented in [6]. On the other hand, the EKF performance depends on the accuracy of the machine model; due to magnetic saturation phenomena, which are significant in traction drives, the position estimation error varies significantly depending on the operating point. The tests show that an EKF with a simple EESM model cannot provide an accurate estimation for different operating points.

\section{HFI-based position estimation}

HFI-based position estimation is tested for speeds lower than $1000 \mathrm{rpm}$. The injected voltage amplitude is $30 \mathrm{~V}$, at 1.5 $\mathrm{kHz}$. A very challenging torque-speed profile is used for the test (see Fig. 8); it includes situations that are not likely to happen in practice.

The pulsating HFI-based self-sensing control seems to be robust enough, especially that no parameters are needed for estimation. It should be noted that the steady state estimation error increases as the speed increases. As for the speed estimation, it seems to be accurate enough.

The dynamical performance of this estimation technique can be improved by fine-tuning the filters and the PLL bandwidths. The EESM under study seems to have advantageous saliency characteristics.

\section{CONCLUDING REMARKS}

The electric vehicle traction drive is a very challenging application for the self-sensing control, because it requires 


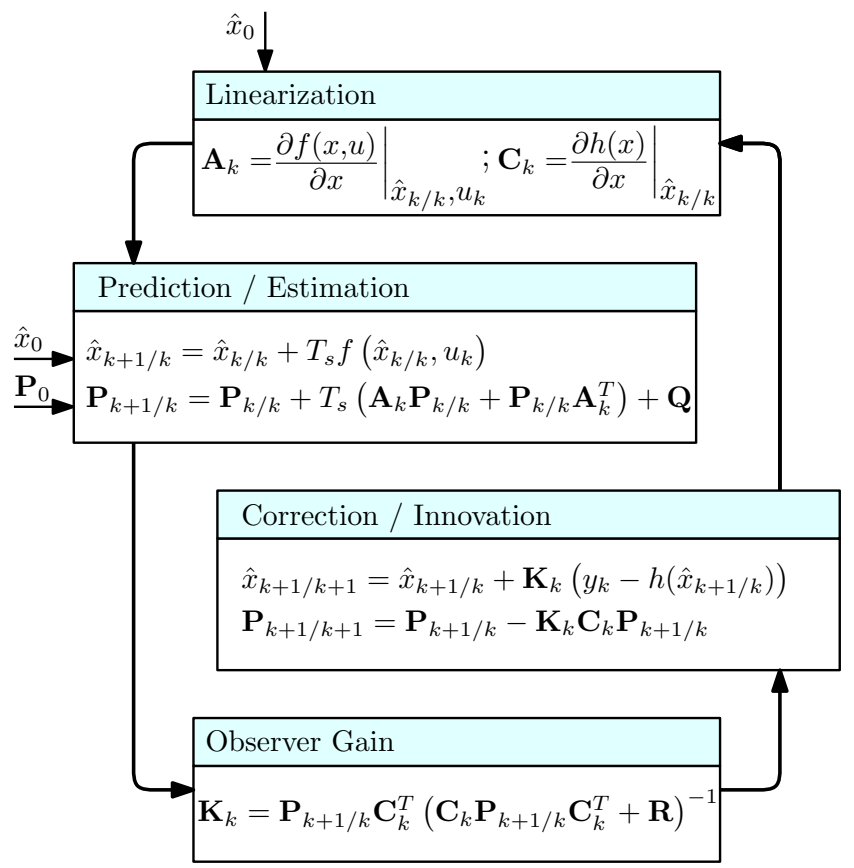

Fig. 5: Extended Kalman Filter estimation algorithm
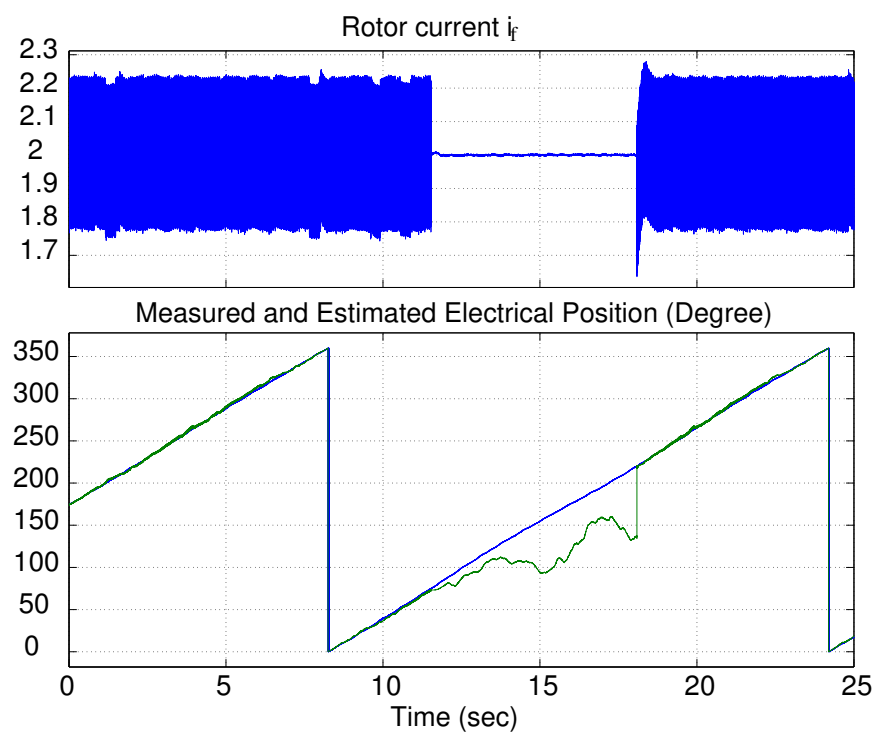

Fig. 6: EKF experimental results.

very accurate position estimation with high dynamical performance, including critical situations such as high-torque zero-speed operation. In addition, the AC machines used in traction have high power-to-volume ratio, with a complex magnetic (saturation) behavior. Throughout this paper, three position estimation approaches have been studied and tested on an EESM traction drive: the equivalent-flux, the stateobserver and the high-frequency injection approaches. The first approach can be applied to all AC machines. Its main limitations are the stability at low speed and the dependence on the accuracy of the inductance $L_{q}$ estimation. The second approach highly relies on the machine inductances, and requires higher computation complexity. The latter approach is accurate and robust enough at low speed, and can be applied for all salient AC machines. However, it suffers from limited dynamical performance at higher speeds. Combining the first (or second) and the latter approaches is a viable estimation strategy that is well known and often used [17]. Further investigations of the rotor winding injection characteristics and benefits [4]-[6] are to be carried out in the future.

\section{REFERENCES}

[1] T. Jahns, "Getting rare-earth magnets out of ev traction machines: A review of the many approaches being pursued to minimize or eliminate rare-earth magnets from future ev drivetrains," IEEE Electrification Magazine, vol. 5, pp. 6-18, March 2017.

[2] J. Holtz, "Sensorless control of induction machines with or without signal injection?," IEEE Transactions on Industrial Electronics, vol. 53 pp. 7-30, 22006.

[3] A. Griffo, D. Drury, T. Sawata, and P. H. Mellor, "Sensorless starting of a wound-field synchronous starter/generator for aerospace applications," IEEE Transactions on Industrial Electronics, vol. 59, pp. 3579-3587, Sept 2012.

[4] J. Choi, I. Jeong, K. Nam, and S. Jung, "Sensorless control for electrically energized synchronous motor based on signal injection to field winding," in IECON 2013 - 39th Annual Conference of the IEEE Industrial Electronics Society, pp. 3120-3129, Nov 2013.

[5] A. Rambetius and B. Piepenbreier, "Carrier signal based sensorless control of wound field synchronous machines using the rotor winding as the receiver: Rotating vs. alternating carrier," in Proceedings of PCIM Europe 2015; International Exhibition and Conference for Power Electronics, Intelligent Motion, Renewable Energy and Energy Management, pp. 1-8, May 2015.

[6] M. Koteich, A. Maloum, G. Duc, and G. Sandou, "Observability analysis of sensorless synchronous machine drives," in 2015 European Control Conference (ECC), pp. 3560-3565, IEEE, 72015.

[7] A. Beciu, E. Godoy, P. Rodriguez-Ayerbe, I. Bahri, and A. Maalouf, "High frequency impedance analysis for sensorless starting of wound rotor synchronous machines," in IFAC 2017, Toulouse, July 2017.

[8] M. Hinkkanen and J. Luomi, "Modified integrator for voltage model flux estimation of induction motors," IEEE Transactions on Industrial Electronics, vol. 50, no. 4, pp. 818-820, 2003.

[9] M. Corley and R. Lorenz, "Rotor position and velocity estimation for a salient-pole permanent magnet synchronous machine at standstill and high speeds," Industry Applications, IEEE Transactions on, vol. 34, no. 4, pp. $784-789,1998$.

[10] J. Holtz, "The representation of ac machine dynamics by complex signal flow graphs," IEEE Transactions on Industrial Electronics, vol. 42, pp. 263-271, 61995.

[11] N. Idris and A. Yatim, "An improved stator flux estimation in steadystate operation for direct torque control of induction machines," IEEE Transactions on Industry Applications, vol. 38, no. 1, pp. 110-116, 2002.

[12] M. Comanescu, L. Xu, and S. Member, "An improved flux observer based on pll frequency estimator for sensorless vector control of induction motors," Industrial Electronics, IEEE Transactions on, vol. 53, no. 1, pp. 50-56, 2005.

[13] I. Boldea, G. D. Andreescu, C. Rossi, A. Pilati, and D. Casadei, "Active flux based motion-sensorless vector control of dc-excited synchronous machines," in Energy Conversion Congress and Exposition, 2009. ECCE 2009. IEEE, pp. 2496-2503, 2009.

[14] M. Koteich, G. Duc, A. Maloum, and G. Sandou, "A unified model for low-cost high-performance ac drives: the equivalent flux concept," in The Third International Conference on Electrical, Electronics, Computer Engineering and their Applications (EECEA), Apr. 2016.

[15] M. Koteich, "Flux estimation algorithms for electric drives: a comparative study," in International Conference on Renewable Energies for Developing Countries 2016 (REDEC2016), July 2016.

[16] L. Harnefors and H.-P. Nee, "A general algorithm for speed and position estimation of ac motors," IEEE Transactions on Industrial Electronics, vol. 47 , no. 1 , pp. $77-83,2000$.

[17] C. Silva, G. M. Asher, and M. Sumner, "Hybrid rotor position observer for wide speed-range sensorless pm motor drives including zero speed,' IEEE Transactions on Industrial Electronics, vol. 53, no. 2, pp. 373-378, 2006. 

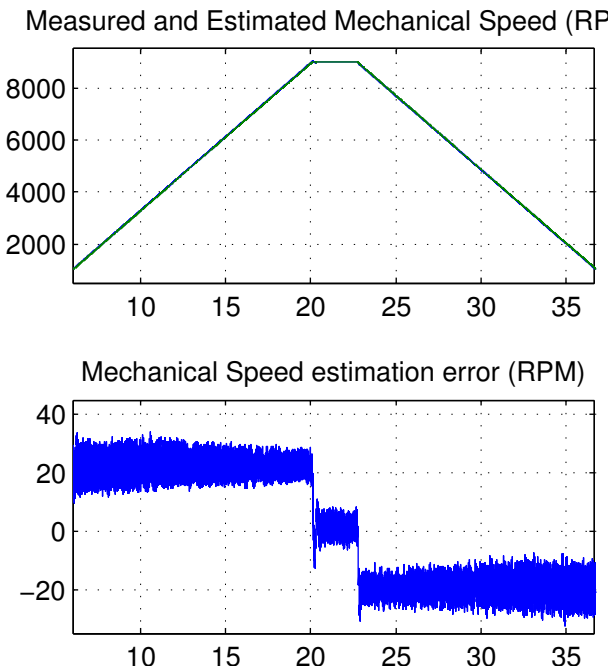

Electrical position estimation error (Degree)

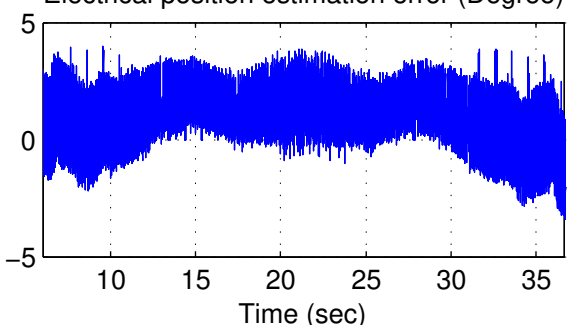

Measured and Estimated Mechanical Speed (RPM)

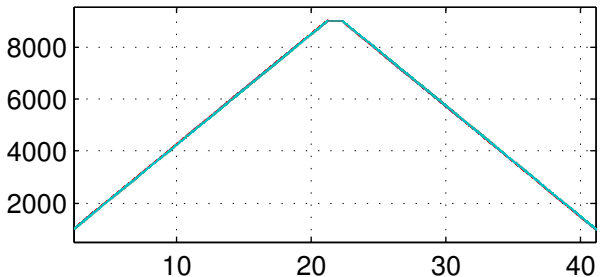

Mechanical Speed estimation error (RPM)

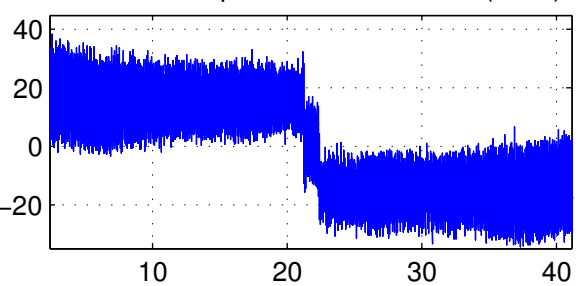

Electrical position estimation error (Degree)

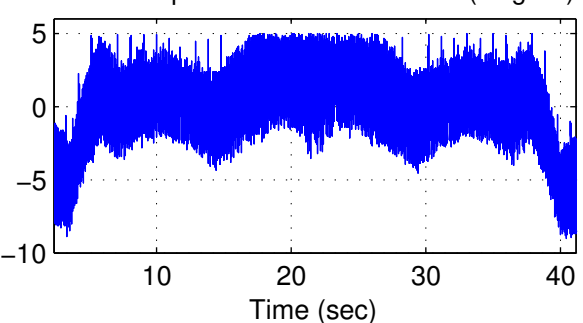

Fig. 7: Flux-based sensorless experimental results: no-load (left) and full-load (right) speed ramp from 1000 to $9000 \mathrm{rpm}$.

Torque (N.m.)

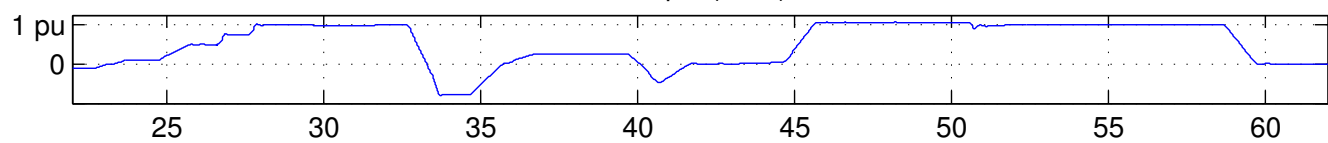

Measured and Estimated Mechanical Speed (RPM)
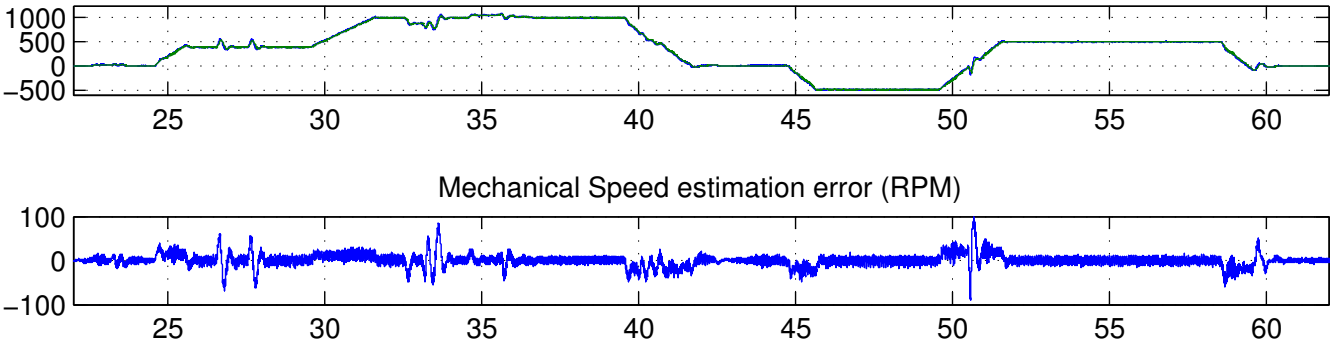

Measured and Estimated Electrical Position (Degree)

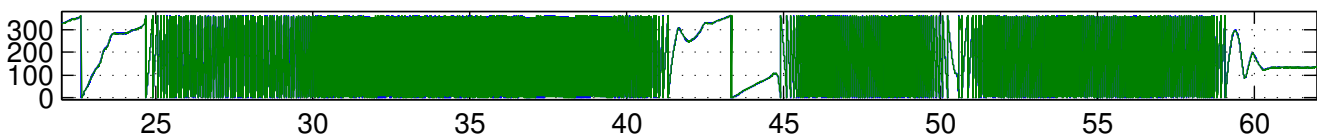

Electrical position estimation error (Degree)

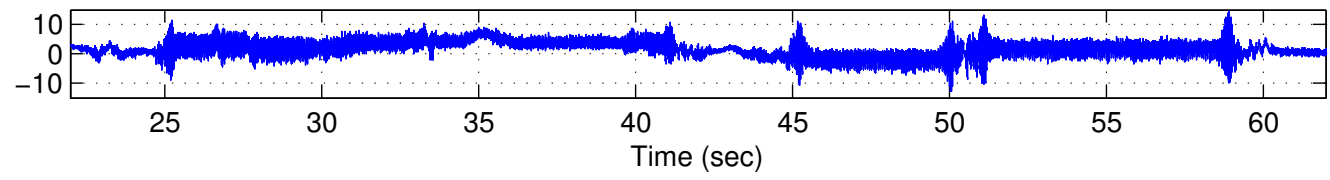

Fig. 8: HFI sensorless experimental results. 